\title{
Tsallis Maximum Entropy Lorenz Curves
}

\author{
M. Yaghoobi Avval Riabi ${ }^{\dagger}$, G. R. Mohtashami Borzadaran ${ }^{\ddagger, *}$ \\ and G. H. Yari ${ }^{\star}$ \\ † Islamic Azad University, Gonabad Branch \\ $\ddagger$ Ferdowsi University of Mashhad \\ * Iran University of Science and Technology
}

\begin{abstract}
In this paper, at first we derive a family of maximum Tsallis entropy distributions under optional side conditions on the mean income and the Gini index. Furthermore, corresponding with these distributions a family of Lorenz curves compatible with the optional side conditions is generated. Meanwhile, we show that our results reduce to Shannon entropy as $\beta$ tends to one. Finally, by using actual data, we compare the maximum Tsallis entropy Lorenz curve with some parametric Lorenz curves.
\end{abstract}

Keywords. Tsallis entropy; Shannon entropy; maximum entropy principle; Lorenz Curve, Gini index; income distribution.

MSC 2010: 62B10; 94A17.

\section{Introduction}

Recently the concept of entropy has been used in many research areas, particularly in economics. In this research area, classical models often consider perfect information which in many cases requires some assumptions to estimate functions. Some new approaches to the treatment of problems in economics are made using methods that exploit the use of entropy. The use of concepts such as entropy is growing over time, giving rise to other concepts and methodologies, such as Maximum Entropy and Cross-Entropy . The application of these methods began with the analysis of financial markets, and

* Corresponding author 
was later extended to other areas such as industrial economics, agricultural economics or microeconomics. (For more ditails see Ferreira and Dionísio, 2012).

Suppose that a random variable has a known probability density function, so, it determines the distribution completely. But in many cases, the explicit density is unknown and must be estimated. The classical procedure consists of fitting an analytical function to the observations. Another more logical approach is to apply the maximum entropy that was proposed by Jaynes (1957). This technique allows choosing among all the possible probability distributions the most suitable one with respect to the available knowledge. The maximum entropy principle states that when some information is given about a random variable, the least biased probability distribution can be obtained by maximizing the Shannon entropy subject to the given constraints. The Shannon entropy is additive, but additivity is not a necessary condition for a function to measure the uncertainty of a probability distribution. Over the past 60 years, after Shannon introduced his measure of entropy, various forms of the entropy have been suggested that were non additive. One of them is the Tsallis entropy, that was introduced by Tsallis (1988). Tsallis entropy is a one-parameter generalization of the Shannon entropy which can lead to models or statistical results different from those obtained by the Shanoon entropy. It should be noted that Tsallis entropy is a monotonic function of Renyi entropy. On the other hand, Tsallis distributions (the ones derived from the maximization of Tsallis entropy) are of great interest in many branches of science since they are similar to generalized Pareto distributions and appear as the limit distribution of excesses over a threshold (see Yaghoobi et al., 2010). A review of Tsallis entropy and its applications are presented in Tsallis (2000).

There are many papers, books, monographs published about maximum Shannon entropy and maximum Tsallis entropy such as Jaynes (1982), Zellner and Highfield (1988), Kapur (1989), Ryu (1993), Dos Santos (1997), Dober and Bolle (2001), Grender and Grender (2001), Harremoes and Topsoe (2001), Wu (2003), Chery and Maslov (2004), Dukkiputi et al. (2005), Karmeshu and Sharma (2006), Cover and Thomas (2006) and Holm (1993). Holm (1993) especially applied maximum Shannon entropy for finding the Lorenz curve.

This paper has two main objectives. First, we derive a family of maximum Tsallis entropy distributions under the conditions on the mean and the Gini index. Afterword, as a consequence of the first objective, we find families of 
Lorenz curves.

\section{Preliminaries}

In this section, we provide some basic definitions that are necessary in the next section.

\subsection{Shannon Entropy and Tsallis Entropy}

The Shannon entropy of a continuous random variable $X$, taking its values in $R$, with probability density function $f(x)$, is defined by

$$
H(f)=-\int_{R} \log f(x) f(x) d x,
$$

provided that, the integral exists.

One extension of the Shannon entropy is the Tsallis entropy which is defined as:

$$
H_{\beta}(f)=\frac{1}{\beta-1}\left\{1-\int_{R} f^{\beta}(x) d x\right\} .
$$

It is easy to show that, $H_{\beta}(f)=H(f)$ as $\beta \longrightarrow 1$. Therefore, the Tsallis entropy is a generalization of the Shannon entropy.

\subsection{The Lorenz Curve}

The Lorenz curve is an important tool for analyzing income distributions introduced by Max Lorenz (1905). He proposes a simple graphical means to summarize the inequality of wealth. Graphically, the Lorenz curves gives the proportion of the total societal income accruing to the lowest earning proportion of income earners.

Definition 1. Let $X$ be a non-negative random variable with probability density function $f_{X}(x)$ and distribution function $F_{X}(x)$. The Lorenz curve of $X$ denoted by $L_{X}(p)$ is defined as:

$$
L_{X}(p)=\frac{1}{\mu} \int_{0}^{p} F_{X}^{-1}(y) d y, \quad 0 \leqslant p \leqslant 1,
$$

where, $F_{X}^{-1}(y)=\inf \left[x: F_{X}(x) \geqslant y\right]$ is the quantail function and $\mu$ is the mean of $X$. From Definition 1, we can show that, $\mathrm{L}$ is a continuous function 
on $[0,1]$, with $L(0)=0$ and $L(1)=1$. Also $L$ is an increasing and convex function of $p$.

There are various ways for the construction of the parametric families of the Lorenz curves. The first way consists of achieving parametric family distribution and substituting them in (3) (For example see Gastwirth, 1971). Note that the explicit distribution is unknown in many cases, therefore it must be estimated by the classical methods such as maximum entropy.

Another way consists of selecting parametric families of curves that satisfy the properties of the Lorenz curves. Some of the most common parametric Lorenz curves are as follows:

\section{- The Kakwani and Podder specification}

The pioneer model was proposed by Kakwani and Podder (1973). The Kakwani - Podder Lorenz curve is specified as:

$$
L(p)=p^{\alpha} e^{-\beta(1-p)}, \quad 0 \leqslant p \leqslant 1, \quad 1<\alpha<2, \quad \beta>0 .
$$

Kakwani and Podder (1976) also proposed a new parametric model based on a geometric motivation.

\section{- The Gupta specification}

Gupta (1984) provided another parametric Lorenz curve in the following form:

$$
L(p)=p \alpha^{p-1}, \quad 0 \leqslant p \leqslant 1, \quad \alpha>0 .
$$

\section{- The Rasche specification}

Rasche et al. (1980) suggested the following form for Lorenz curve,

$$
L(p)=\left[1-(1-p)^{\alpha}\right]^{\frac{1}{\beta}}, \quad 0 \leqslant p \leqslant 1, \quad 0<\beta, \quad 0<\alpha \leqslant 1 .
$$

If $\beta=1$ and $\alpha<1$, we obtain the Lorenz curve corresponding to the classical Pareto distribution with the form:

$$
L(p)=1-(1-p)^{\alpha}, \quad 0 \leqslant p \leqslant 1, \quad 0<\alpha \leqslant 1 .
$$




\section{- The Chotikopanich specification}

The Chotikopanich Lorenz curve was proposed by Chotikopanich (1993). This model is as follows:

$$
L(p)=\frac{e^{k p}-1}{e^{k}-1}, \quad 0 \leqslant p \leqslant 1, \quad k>0 .
$$

\section{- The Pakes specification}

The family proposed by Pakes (1981) has a Lorenz curve of the form:

$$
L(p)=\frac{\int_{0}^{p} x^{\alpha-1}(1-x)^{\beta-1}}{B(\alpha, \beta)} d x, \quad 0 \leqslant p \leqslant 1,
$$

where $\alpha \geqslant 1, \quad 0<\beta \leqslant 1$ and $B$ is the beta function.

For more details, see for example: Kleiber (2005), Kleiber and Kotz (2003), Sarabia (2008), Arnold (2007), Villasenor and Arnold (1989) and Rohde (2008).

\subsection{Gini Index}

The Gini index was developed by Corrado Gini (1912). It is strictly connected with representation of income inequality via the Lorenz curve. The Gini index is given as twice the area between the Lorenz curve and the equality line.

Definition 2. Let $X$ be a non-negative random variable with Lorenz curve, $L(p)$. The Gini index is denoted by $G$ and is defined as:

$$
G=2 \int_{0}^{1}\{p-L(p)\} d p=1-2 \int_{0}^{1} L(p) d p,
$$

we note that, $0 \leqslant G \leqslant 1$.

By setting the $L(p)$ of (3) in (4), the following useful form is obtained:

$$
G=\frac{1}{\mu} \int_{0}^{1}(2 p-1) F^{-1}(p) d p .
$$

Note that, the mean income can be written as:

$$
\mu=\int_{0}^{1} F^{-1}(p) d p .
$$


For more details, see Gastwirth (1971) and Nembua (2006).

Usually the Gini index is calculated from the Lorenz curve, but we shall do the opposite.

\section{Main Results}

Let $X$ be a non-negative and absoulutely continuous random variable ${ }^{1}$ indicating the level of income, with values in the interval $\left[x_{0}, x_{1}\right]$. Also, suppose $f(x)$ and $F(x)$ are the probability density function and the distribution function of $X$, respectively. Furthermore, assume $F(x)$ is strictly increasing. Tsallis entropy of this distribution is defined as:

$$
H_{\beta}(f)=\frac{1}{\beta-1}\left\{1-\int_{x_{0}}^{x_{1}} f^{\beta}(x) d x\right\}
$$

by choosing $F(x)=p$, we have:

$$
x=F^{-1}(p) \Longrightarrow f(x)=f\left(F^{-1}(p)\right) .
$$

On the other hand, we note that $F\left(F^{-1}(p)\right)=p$, so

$$
\frac{d F^{-1}(p)}{d p} f\left(F^{-1}(p)\right)=1, \quad \text { (chain rule) }
$$

therfore,

$$
f\left(F^{-1}(p)\right)=\frac{1}{\frac{d F^{-1}(p)}{d p}} .
$$

Finally, by substituting above relations into (7) we derive:

$$
H_{\beta}(f)=\frac{1}{\beta-1}\left\{1-\int_{0}^{1}\left(\frac{d F^{-1}}{d p}\right)^{1-\beta} d p\right\},
$$

where, $F^{-1}$ is the inverse function of $F$.

We intend to obtain the maximum of the function described in (7), provided that the mean and the Gini index are known.

On the other hand, via integration by part it is easy to show that, the relations (5) and (6) are equal to,

$$
\int_{0}^{1}(1-p) \frac{d F^{-1}}{d p} d p=\mu-x_{0}
$$




$$
\int_{0}^{1}(1-p) p \frac{d F^{-1}}{d p} d p=G \mu
$$

respectively.

Now, we consider the following optimization problem,

$$
\max H_{\beta}(f)=\frac{1}{\beta-1}\left\{1-\int_{0}^{1}\left(\frac{d F^{-1}}{d p}\right)^{1-\beta} d p\right\},
$$

such that,

$$
\begin{gathered}
\int_{0}^{1}(1-p) \frac{d F^{-1}}{d p} d p=\mu-x_{0}, \\
\int_{0}^{1}(1-p) p \frac{d F^{-1}}{d p} d p=G \mu .
\end{gathered}
$$

For using the Lagrangian method we define $\Lambda(f)$ as:

$$
\begin{aligned}
\Lambda(f)= & \frac{1}{\beta-1}\left\{1-\int_{0}^{1}\left(\frac{d F^{-1}}{d p}\right)^{1-\beta} d p\right\} \\
& -\lambda_{1}\left\{\int_{0}^{1}(1-p) \frac{d F^{-1}}{d p} d p-\left(\mu-x_{0}\right)\right\} \\
& -\lambda_{2}\left\{\int_{0}^{1}(1-p) p \frac{d F^{-1}}{d p} d p-G \mu\right\},
\end{aligned}
$$

by differentiating $\Lambda(f)$ and some calculations we obtain:

$$
\frac{d F^{-1}}{d p}=\frac{1}{\left\{\lambda_{1}(1-p)+\lambda_{2} p(1-p)\right\}^{\frac{1}{\beta}}},
$$

in which, $\lambda_{1}$ and $\lambda_{2}$ are the undetermined Lagrange multipliers arising from the condition (9) and (10), respectively. Various values of $\beta, \quad \lambda_{1}$ and $\lambda_{2}$ lead to familiar distributions, some of which are discussed in the next subsections.

\subsection{Maximum Tsallis Entropy Lorenz Curve}

A family of Lorenz curves that is in agreement with the conditions will be generated from (11). For all members of this family, we have $L(0)=0$, $L(1)=1$. The different cases are classified below: 


\subsection{1 $\lambda_{1} \neq 0$ and $\lambda_{2}=0$}

In this case, equation (11) reduces to

$$
\frac{d F^{-1}}{d p}=\frac{(1-p)^{-\frac{1}{\beta}}}{\sqrt[\beta]{\lambda_{1}}}
$$

An integration of $\frac{d F^{-1}}{d p}$ over $[0, p]$, we obtain

$$
F^{-1}(p)=\frac{\beta}{\beta-1} \cdot \frac{1}{\sqrt[\beta]{\lambda_{1}}}\left\{1-(1-p)^{1-\frac{1}{\beta}}\right\}+x_{0} .
$$

Now by using (5) and (6) we derive:

$$
x_{0}=\left\{1-\left(3-\frac{1}{\beta}\right) G\right\} \mu,
$$

and so,

$$
F^{-1}(p)=\mu\left[1+G \frac{3 \beta-1}{\beta-1}\left\{1-\frac{(3 \beta-1)(2 \beta-1)}{\beta}(1-p)^{1-\frac{1}{\beta}}\right\}\right] .
$$

Since $F^{-1}(p)=x$, then $F(x)=p$, by some calculations we derive:

$$
F(x)=1-\left\{\frac{\beta}{(3 \beta-1)(2 \beta-1)}+\frac{x-\mu}{G \mu \frac{(3 \beta-1)^{2}(2 \beta-1)}{\beta(1-\beta)}}\right\}^{-\left(\frac{\beta}{1-\beta}\right)}
$$

Finally substituting $F^{-1}$ into (3) gives a one-parametric model:

$$
L_{*}^{\beta}(p)=p+G \frac{3 \beta-1}{\beta-1}\left\{p-1+(1-p)^{2-\frac{1}{\beta}}\right\},
$$

where, $\beta$ may be used for optimization with respect to additional information.

- The Pareto distributions provide models for many applications in social science, physical science and economics. Also, this family is related to many other families of distributions. The cumulative distribution of Pareto $(I V)(\mu, \theta, \gamma, \alpha)$ is as follows:

$$
F(x)=1-\left\{1+\left(\frac{x-\mu}{\theta}\right)^{\frac{1}{\gamma}}\right\}^{-\alpha}, \quad x>\mu .
$$




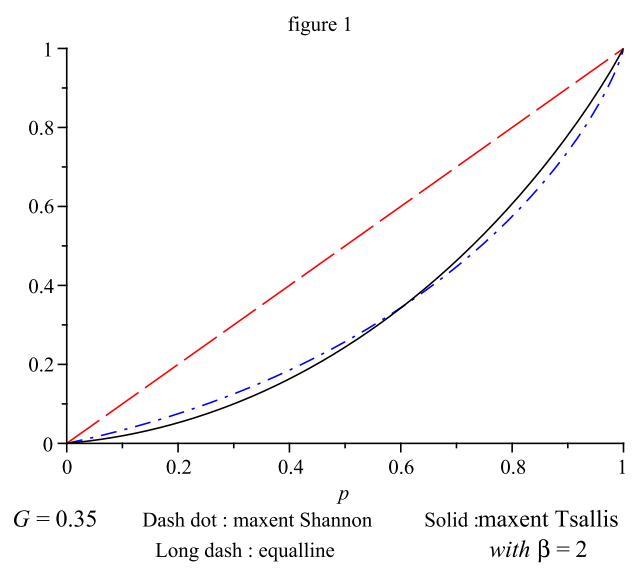

Putting $(\alpha=1),(\gamma=1)$ and $(\gamma=1, \mu=\theta)$ in the above relation leads us to the cumulative distribution function of Pareto (III), Pareto (II) and Pareto (I), respectively.

Comparing (14) and (16), we find that, if we set $\beta=\frac{3+\sqrt{3}}{6}$, in (14), it reduces to a cumulative distribution function of Pareto $(I I)(\mu,(3+2 \sqrt{3}) G \mu,(2+$ $\sqrt{3})$ ), and if we set $\beta=\frac{3+\sqrt{3}}{6}$, in (15), we obtain the Lorenz curve corresponding to Pareto $(I I)(\mu,(3+2 \sqrt{3}) G \mu,(2+\sqrt{3}))$.

Further, if we set $\beta=\frac{3+\sqrt{3}}{6}$ and $G=\frac{1}{3+2 \sqrt{3}}$ in (14) and (15), then we obtain the distribution function of Pareto $(I)(\mu,(2+\sqrt{3}))$ and its Lorenz curve respectively.

- A predicted minimum income $x_{0}=0$, the standard lower bound of many income distributions, is obtained for $G=\frac{\beta}{3 \beta-1}$.

- Note that in $(15), \lim _{\beta \longrightarrow 1} L_{*}^{\beta}(p)$ gives the function that was obtained by Holm (1993). In this case

$$
L_{*}(p)=p+2 G(1-p) \ln (1-p)
$$

Figure 1 shows two Lorenz curves corresponding with model (17) and model (15) with $\beta=2$, when $G=0.35$. 


\subsection{2 $\lambda_{1}=0$ and $\lambda_{2} \neq 0$}

Equation (11) with $\lambda_{1}=0, \lambda_{2} \neq 0$ implies a density quantile function symmetric with respect to $p=0.5$. By integration of $\frac{d F^{-1}}{d p}$ over $[0, p]$, we obtain:

$$
F^{-1}(p)=\frac{1}{\sqrt[\beta]{\lambda_{2}}} \int_{0}^{p} t^{-\frac{1}{\beta}}(1-t)^{-\frac{1}{\beta}} d t+x_{0}
$$

considering an expansion of $(1-t)^{-\frac{1}{\beta}}=\sum_{j=0}^{\infty}\left(\begin{array}{c}-\frac{1}{\beta} \\ j\end{array}\right)(-1)^{j} t^{j}$, we are led to:

$$
F^{-1}(p)=\frac{1}{\sqrt[\beta]{\lambda_{2}}} \sum_{j=0}^{\infty}\left(\begin{array}{c}
-\frac{1}{\beta} \\
j
\end{array}\right)(-1)^{j} \frac{p^{j-\frac{1}{\beta}+1}}{j-\frac{1}{\beta}+1}+x_{0} .
$$

Now by using (9) and (10) we derive:

$$
x_{0}=\mu\left\{1-G \frac{B\left(1-\frac{1}{\beta}, 2-\frac{1}{\beta}\right)}{B\left(2-\frac{1}{\beta}, 2-\frac{1}{\beta}\right)}\right\} .
$$

Finally, by substitution of $F^{-1}$ into (3) we obtain:

$$
L_{*}^{\beta}(p)=p+G\left\{\frac{2-3 \beta}{\beta-1} p+\sum_{j=0}^{\infty} \frac{\left(\begin{array}{c}
-\frac{1}{\beta} \\
j
\end{array}\right)(-1)^{j} \frac{p^{j-\frac{1}{\beta}+2}}{\left(j-\frac{1}{\beta}+2\right)\left(j-\frac{1}{\beta}+1\right)}}{B\left(2-\frac{1}{\beta}, 2-\frac{1}{\beta}\right)}\right\},
$$

in which, $B(a, b)=\int_{0}^{1} t^{a-1}(1-t)^{b-1} d t$.

- Note that $\lim _{\beta \longrightarrow 1} L_{*}^{\beta}(p)=p+G[p \ln (p)+(1-p) \ln (1-p)]$ that was attained by Holm (1993).

\subsection{3 $\lambda_{1} \neq 0$ and $\lambda_{2} \neq 0$}

Equation (11) with $\lambda_{1} \neq 0, \lambda_{2} \neq 0$ represents the following complex model,

$$
\frac{d F^{-1}}{d p}=\frac{1}{\left\{\lambda_{1}(1-p)+\lambda_{2} p(1-p)\right\}^{\frac{1}{\beta}}}=\frac{1}{\sqrt[\beta]{\lambda_{1}}}(1-p)^{-\frac{1}{\beta}}(1+a p)^{-\frac{1}{\beta}},
$$

where $a=\frac{\lambda_{2}}{\lambda_{1}}$.

With a similar process, integration over $[0, p]$ gives

$$
F^{-1}(p)=\frac{1}{\sqrt[\beta]{\lambda_{1}}} \int_{0}^{p}(1-t)^{-\frac{1}{\beta}}(1+a t)^{-\frac{1}{\beta}} d t+x_{0}
$$


We know that, $(1+x)^{s}=\sum_{m=0}^{\infty} A(s, m) x^{m}$ and $(1-x)^{s}=\sum_{m=0}^{\infty}(-1)^{m}$ $\times A(s, m) x^{m}$ in which, $A(s, m)=\frac{\Gamma(s+1)}{\Gamma(s+1-m) \Gamma(m+1)}$.

By using the above expansion for $(1+a t)^{-\frac{1}{\beta}}$ and $(1-t)^{-\frac{1}{\beta}}$ we derive:

$$
\begin{aligned}
F^{-1}(p)= & \frac{1}{\sqrt[\beta]{\lambda_{1}}} \sum_{n=0}^{\infty} \sum_{m=0}^{\infty}(-1)^{n} a^{m} A\left(-\frac{1}{\beta}, n\right) A\left(-\frac{1}{\beta}, m\right) \\
& \times \int_{0}^{p} \frac{t^{m+n+1}}{m+n+1} d t+x_{0}, \quad a<1 . \\
F^{-1}(p)= & \frac{1}{\sqrt[\beta]{\lambda_{1}}} \sum_{n=0}^{\infty} \sum_{m=0}^{\infty}(-1)^{n} a^{m} A\left(-\frac{1}{\beta}, n\right) A\left(-\frac{1}{\beta}, m\right) \\
& \times \frac{p^{m+n+1}}{m+n+1}+x_{0}, \quad a<1 .
\end{aligned}
$$

With a common procedure we have the following results for the twoparameter model:

$$
\begin{aligned}
L_{*}^{\beta}(p)= & p-G \frac{\sum_{m=0}^{\infty} A\left(-\frac{1}{\beta}, m\right) a^{m} B\left(m+1,2-\frac{1}{\beta}\right)}{\sum_{m=0}^{\infty} A\left(-\frac{1}{\beta}, m\right) a^{m} B\left(m+2,2-\frac{1}{\beta}\right)} p \\
& +G \frac{\left\{\sum_{n=0}^{\infty} \sum_{m=0}^{\infty}(-1)^{n} a^{m} A\left(-\frac{1}{\beta}, n\right) A\left(-\frac{1}{\beta}, m\right)\right\} \frac{p^{m+n+2}}{(m+n+1)(m+n+2)}}{\sum_{m=0}^{\infty} A\left(-\frac{1}{\beta}, m\right) a^{m} B\left(m+2,2-\frac{1}{\beta}\right)} .
\end{aligned}
$$

\subsection{An Application and Some Comparisons}

For using a specific model it must pass the test of empirical data. In this study the maximum Tsallis entropy model (15) is evaluated by examining the residual vector $R_{i}=L\left(p_{i}\right)-L_{i}$ for an actual data set $\left\{\left(p_{i} L_{i}\right) \mid i=\right.$ $1,2, \ldots, 9\}$ given in Table 2 of Villasenor and Arnold (1989) with an actual value $G=0.3196$.

From Table 1, we note that $\beta=1.3$ is the minimum sum square error (SSE), therefore to compare $\hat{L}^{\beta}$ with other models, we use $\hat{L}^{1.3}$ in Table 2 . 
Table 1. Maximum Tsallis Entropy model (15) for some $\beta$ values

\begin{tabular}{cccccccccc}
\hline \hline$p$ & $L$ & $\hat{L}_{*}^{0.9}$ & $\hat{L}_{*}^{1.1}$ & $\hat{L}_{*}^{1.2}$ & $\hat{\boldsymbol{L}}_{*}^{\mathbf{1 . 3}}$ & $\hat{L}_{*}^{1.4}$ & $\hat{L}_{*}^{1.5}$ & $\hat{L}_{*}^{1.7}$ & $\hat{L}_{*}^{1.9}$ \\
\hline 0.1 & 0.0213 & 0.04242 & 0.03693 & 0.03491 & $\mathbf{0 . 0 3 3 2 1}$ & 0.03176 & 0.03051 & 0.02847 & 0.02687 \\
0.2 & 0.0657 & 0.09089 & 0.08191 & 0.07865 & $\mathbf{0 . 0 7 5 9 5}$ & 0.07366 & 0.07171 & 0.06853 & 0.06607 \\
0.3 & 0.1273 & 0.14625 & 0.13583 & 0.13215 & $\mathbf{0 . 1 2 9 1 2}$ & 0.12659 & 0.12445 & 0.12102 & 0.11838 \\
0.4 & 0.2001 & 0.20962 & 0.19987 & 0.19654 & $\mathbf{0 . 1 9 3 8 7}$ & 0.19167 & 0.18984 & 0.18694 & 0.18477 \\
0.5 & 0.2833 & 0.28251 & 0.27555 & 0.27335 & $\mathbf{0 . 2 7 1 6 6}$ & 0.27032 & 0.26923 & 0.26760 & 0.26644 \\
0.6 & 0.3781 & 0.36701 & 0.36499 & 0.36463 & $\mathbf{0 . 3 6 4 4 7}$ & 0.36443 & 0.36447 & 0.36467 & 0.36493 \\
0.7 & 0.4867 & 0.46669 & 0.47137 & 0.47338 & $\mathbf{0 . 4 7 5 1 7}$ & 0.47674 & 0.47814 & 0.48048 & 0.48237 \\
0.8 & 0.6119 & 0.58722 & 0.59989 & 0.60449 & $\mathbf{0 . 6 0 8 3 1}$ & 0.61151 & 0.61422 & 0.61859 & 0.62194 \\
0.9 & 0.7624 & 0.74159 & 0.76117 & 0.76758 & $\mathbf{0 . 7 7 2 6 5}$ & 0.77675 & 0.78012 & 0.78534 & 0.78917 \\
\hline$S S E$ & & 0.00309 & 0.00118 & 0.00092 & $\mathbf{0 . 0 0 0 8 6}$ & 0.00090 & 0.001 & 0.0013 & 0.0016 \\
\hline
\end{tabular}

$L$ : actual value ${\hat{L_{*}}}^{\beta}$ : Maximum Tsallis Entropy model $(15)$ with $\beta=0.9,1.1, \ldots, 1.9$

Table 2. Actual and estimated values for Lorenz curve

\begin{tabular}{cccccc}
\hline \hline$p$ & $L$ & $\hat{L}_{K P}$ & $\hat{L}_{P}$ & $\hat{L}_{C L}$ & $\hat{L}_{*}^{1.3}$ \\
\hline 0.1 & 0.0213 & 0.02198 & 0.0331 & 0.05961 & $\mathbf{0 . 0 3 3 2 1}$ \\
0.2 & 0.0657 & 0.06368 & 0.0843 & 0.12206 & $\mathbf{0 . 0 7 5 9 5}$ \\
0.3 & 0.1273 & 0.12112 & 0.1470 & 0.18785 & $\mathbf{0 . 1 2 9 1 2}$ \\
0.4 & 0.2001 & 0.19393 & 0.2200 & 0.25769 & $\mathbf{0 . 1 9 3 8 7}$ \\
0.5 & 0.2833 & 0.28255 & 0.3025 & 0.33259 & $\mathbf{0 . 2 7 1 6 6}$ \\
0.6 & 0.3781 & 0.38781 & 0.3958 & 0.41405 & $\mathbf{0 . 3 6 4 4 7}$ \\
0.7 & 0.4867 & 0.51080 & 0.5013 & 0.50458 & $\mathbf{0 . 4 7 5 1 7}$ \\
0.8 & 0.6119 & 0.65283 & 0.6225 & 0.60893 & $\mathbf{0 . 6 0 8 3 1}$ \\
0.9 & 0.7624 & 0.81535 & 0.7683 & 0.73900 & $\mathbf{0 . 7 7 2 6 5}$ \\
\hline$S S E$ & & 0.00523 & 0.00231 & 0.01622 & $\mathbf{0 . 0 0 0 8 6}$ \\
$S A E$ & & 0.14349 & 0.138 & 0.3423 & $\mathbf{0 . 0 8 0 8}$ \\
\hline
\end{tabular}

$L$ : actual value $\hat{L}_{k p}$ : Kakwani - Podder model $\hat{L}_{p}$ : peak model

$\hat{L}_{c l}$ : classical Pareto model ${\hat{L_{*}}}^{1.3}$ : Maximum Tsallis Entropy model (15).

Table 2 consists of the actual values $L$, estimated values via KakwaniPodder $\hat{L}_{K P}$, with $\alpha=1.462$ and $\beta=0.501$, Pakes $\hat{L}_{P}$ with $\alpha=1.33$ and $\beta=0.727$, and classic Pareto $\hat{L}_{C L}$ with $\alpha=0.583$ that have been obtained by Villasenor and Arnold (1989), also the obtained values from maximum Tsallis entropy model (15) $\hat{L}_{*}^{\beta}$, with $\beta=1.3^{2}$. 


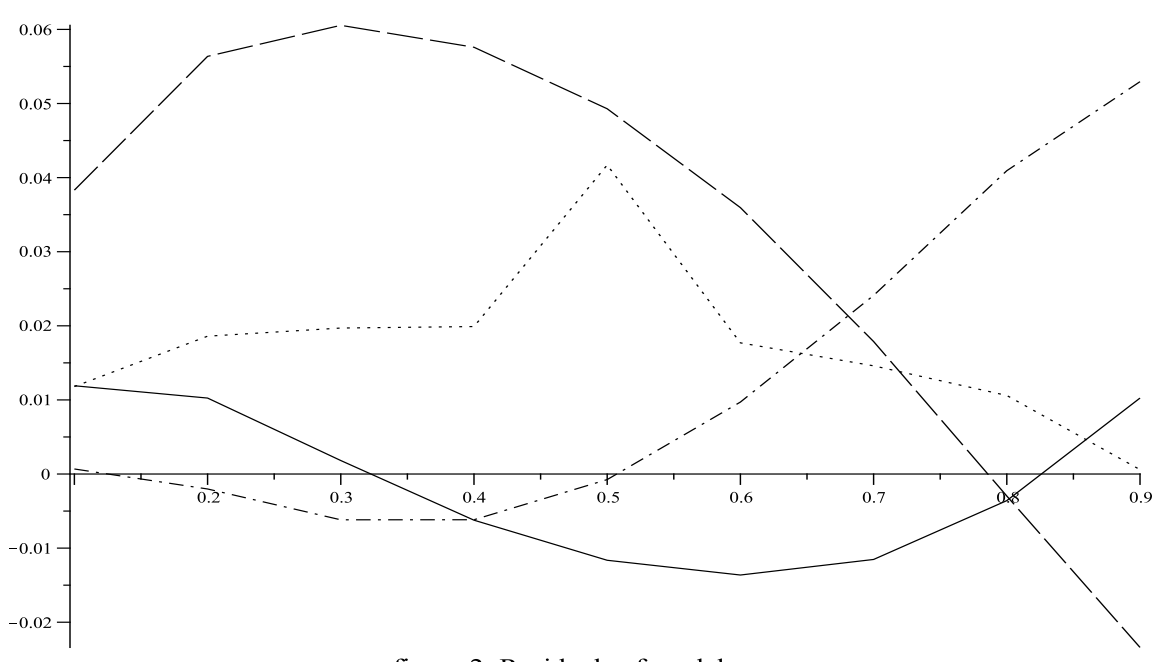

figure 2: Residuals of models

Solid: MaxTsallisEnt Eq: (15)

Dash-Dot:Kakwani-Podder model
Long-Dash:classic pareto model

Dot: Peak model

Furthermore, Figure 2 displays residual vectors of Kakwani-Podder model with $\alpha=1.462$ and $\beta=0.501$, Pakes model with $\alpha=1.33$ and $\beta=0.727$, classic Pareto model with $\alpha=0.583$, and maximum Tsallis entropy model (15) with $\beta=1.3$.

We observe in Table 2 that $\hat{L}^{1.3}$ is improved upon by $\hat{L}_{K P}, \hat{L}_{P}$ and $\hat{L}_{C L}$ on the basis of sum of square errors (SSE) and sum of absolute errors (SAE) ${ }^{3}$ (as the goodness of fit criteria).

\section{Conclusions}

In this study, the maximum Tsallis entropy density function of an income distribution was found, when the mean and the Gini index are known and as a consequence of this process, several models for Lorenz curve have been obtained. We have shown that our findings in $\beta \rightarrow 1$ are compatible with the findings of Shannon entropy which some of them have been achieved by Holm (1993). 


\section{Acknowledgement}

The authors are grateful to the editor and the referees for their helpful comments, which have greatly improved the paper.

\section{Descriptions}

1. Its cumulative distribution function is continuous and differentiable.

2. The last column in Table 2 is our work and other columns are obtained by Villasenor and Arnold (1989).

3. Note that for observations $x_{1}, x_{2}, \ldots, x_{n}$ we have:

$S S E=\sum_{i=0}^{n}\left(x_{i}-\hat{x}\right)^{2}$

$S A E=\sum_{i=0}^{n}\left|x_{i}-\hat{x}\right|$

where $\hat{x}_{i}$ is estimate of $x_{i}$.

\section{References}

Arnold, B.C. (2007). The Lorenz Curve: Evergreen After 100 Years, Advances in Income Inequality Concentration Measures. Routledge, New York.

Cherney, A.S. and Maslov, V.P. (2004). On Minimization and Maximization of Entropy in Various Disciplines. SIAM Journal of Theory of Probability and its Applications, 48, 447-464.

Chotikapanich, D. (1993). A Comparison of Alternative Functional Forms for the Lorenz Curve, Economic Letters, 41, 129-138.

Cover, T. and Thomas, J. (2006). Elements of Information Theory. Wiley, New York.

Dobre M. and Bolle L. (2001). Tsallis Generalized Entropy and Maximum Entropy Formalism for Spray Drops Size Distribution Function Prediction, ILASS-Europe 2001, Zurich.

Dos Santos, R.J.V. (1997). Generalization of Shannon's Theorem for Tsallis Entropy. Journal of Mathematical Physics, 38, 4104-4107.

Dukkipati, A., Bhatnagar, S. and Murty, M.N. (2005). Information Theoretic Justification of Boltzmann Selection and Its Generalization to Tsallis Case. Proc. IEEE, 2, 1667-1674.

Ferreira, P. and Dionisio, A. (2012). An Application of General Maximum Entropy to Utility. CEFAGE-UE Working Papers 2012/18. 
Gastwirth, J.L. (1971). A General Definition of the Lorenz Curve, Econometrica, 39, 10371039 .

Gerendar jr, M. and Gerendar, M. (2001). Maximum Entropy: Clearing up Mysteries. Entropy, 3, 58-63.

Gini, C. (1912). Variabilita e Mutabilita. Bologna, Italy.

Gupta, M.R. (1984). Functional Form for Estimating the Lorenz Curve, Econometrica, 52, 1313-1314.

Harremoes, P. and Topsoe, F. (2001). Maximum Entropy Fundamentals. Entropy, 3, 191-226.

Holm, J. (1993). Maximum Entropy Lorenz Curves. Journal of Econometrics, 59, 377-389.

Jaynes, E.T. (1982). On the Rationale of Maximum-entropy Methods. Proc. IEEE, 70, 939952.

Jaynes, E.T. (1957). Information Theory and Statistical Mechanics, Phys. Rev., 106, 620-630.

Kakwani, N. and Podder, N. (1973). On Estimation of Lorenz Curves from Grouped Observations, International Economic Review, 14, 278-292.

Kakwani, T. and Podder, N. (1976). Efficient Estimation of the Lorenz Curve and Associated Inequality Measures from Grouped Observations, Econometrica, 44, 137-149.

Kapur, J.N. (1989). Maximum Entropy Models in Science and Engineering, John Willey, New York.

Karmeshu, and Sharma, S. (2006). Queue Length Distribution of Network Packet Traffic: Tsallis Entropy Maximization with Fractional Moments. IEEE Communications Letters, 10, 34-36.

Kleiber, Ch. and Kotz, S. (2003). Statistical Size Distributions in Economics and Actuarial Sciences. John Wiley.

Kleiber, Ch. (2005). The Lorenz Curve in Economics and Econometrics. Invited paper, GiniLorenz Centennial Conference, Siena.

Lorenz, M.O. (1905). Mothods of Measuring the Concentration of Wealth. Quarterly Publications of the American Statistical Association, 9, 209-219

Nembua, C.Ch. (2006). Linking Gini to Entropy: Measuring Inequality by an Intersonal Class of Indices. Economics Bulletin, 4, 1-9.

Pakes, A.G. (1981). On Income Distributions and Their Lorenz Curves, Tech. rep., Department of Mathematics, University of Western Australia. 
Rasche, R.H., Gaffney, J., Koo, A.Y.C. and Obst, N. (1980). Functional Forms for Estimating the Lorenz Curve, Econometrica, 48, 1061-1062.

Rohde, N. (2008). Modeling Income Distributions and Lorenz Curves. Springer. Chapter 15, 271-283.

Ryu, H.K. (1993). Maximum Entropy Estimation of Density and Regression Functions. Journal of Econometrics, 56, 397-440.

Sarabia, J. (2008). Parametric Lorenz cCrves: Models and Applications. In: Chotikapanich, D. (Ed.), Series: Economic studies in inequality, social exclusion and well-being, 4.

Tsallis, C. (1988). Possible Generalization of Boltzmann-Gibbs Statistics. J. Stat. Phys., 52, 479.

Tsallis, C. (2000). Entropic Nonextensivity: A Possible Measure of Complexity, Opening talk delivered at the International Workshop on Classical and Quantum Complexity and Nonextensive Thermodynamics, held in Denton-Texas.

Villasenor, J.A. and Arnold, B.C. (1989). Elliptical Lorenz Curves, Journal of Econometrics, 40, 327-338.

Wu, X. (2003). Calculation of Maximum Entropy Densities with Application to Income Distribution. Journal of Econometrics, 115, 347-354.

Yaghoobi Avval Riabi, M., Mohtashami Borzadaran, G.R. and Yari, G.H. (2010). $\beta$-entropy for Pareto-type Distributions and Related Weighted Distributions. Statistics and Probability Letters, 80, 1512-1519.

Zellner, A. and Highfield, R.A. (1988). Calculation of Maximum Entropy Distributions and Approximation of Marginalposterior Distributions. Journal of Econometrics, 37, 195-209.

\section{Yaghoobi Avval Riabi}

Department of Statistics, Islamic Azad University,

Gonabad Branch,

Gonabad, Iran.

email:m_yagoobiawal@yahoo.com

\author{
G. Mohtashami Morzadaran \\ Department of Statistics, \\ Ferdowsi University of Mashhad, \\ Mashhad, Iran. \\ email: gmb1334@yahoo.com
}

\section{G. Yari}

Department of Statistics,

Iran University of Science and Technology,

Tehran, Iran.

email:yari@iust.ac.ir 